\title{
TRANSITION METAL-RELATED CENTRES IN SILICON STUDIED BY HIGH-RESOLUTION DEEP LEVEL TRANSIENT SPECTROSCOPY*
}

\author{
L. DobACzeWSKI ${ }^{a}$, P. KAMIŃski ${ }^{b}$, R. Kozeowski ${ }^{b}$ AND M. SURMA ${ }^{a}$ \\ ${ }^{a}$ Institute of Physics, Polish Academy of Sciences \\ Al. Lotników 32/46, 02-668 Warszawa, Poland \\ ${ }^{b}$ Institute of Electronic Materials Technology \\ Wólczyńska 133, 01-919 Warszawa, Poland
}

\begin{abstract}
High-resolution Laplace-transform deep level transient spectroscopy technique has been used to study a fine structure in the carrier emission process for transition metal- and thermal donors-related defects in silicon. For the case of the transition metal centres the method revealed the fine structure when the defect has a similar emission characteristics to other defects in the crystal. The method also demonstrated the complex emission process for the thermal donors.
\end{abstract}

PACS numbers: 68.55.Ln, 71.55.Cn, 73.40.Lq

Transition metal (TM) impurities are among the most common contaminants of silicon and they have received growing interest from a point of view of high quality Si-crystal production. Besides an initial residual contamination of as-grown crystals, these metals influence the device performance if introduced unintentionally during the silicon crystallization process. The deep level transient spectroscopy (DLTS) is generally used for the TM detection and TM-related defect characterization. However, the problem of the DLTS technique is such that, although it allows to observe defects with very low concentrations, it offers too low resolution to distinguish defects having very similar emission characteristics. A lack of a proper distinction between the intentional and unintentional defects and the emission characteristics coincidence between transition metal- and other defect-related peaks can be the reason for some scatter in the transition metal defect parameters reported in the literature. The problem of the insufficient DLTS technique resolution is even more severe for the case of the thermal donors in the $n$-type silicon where it has been already well established that these defects form a family of defects.

*This work is supported by the State Committee for Scientific Research (Republic of Poland) grant No. 8 S501 03505 and the EC grant No. CIPA-CT94-0172. 


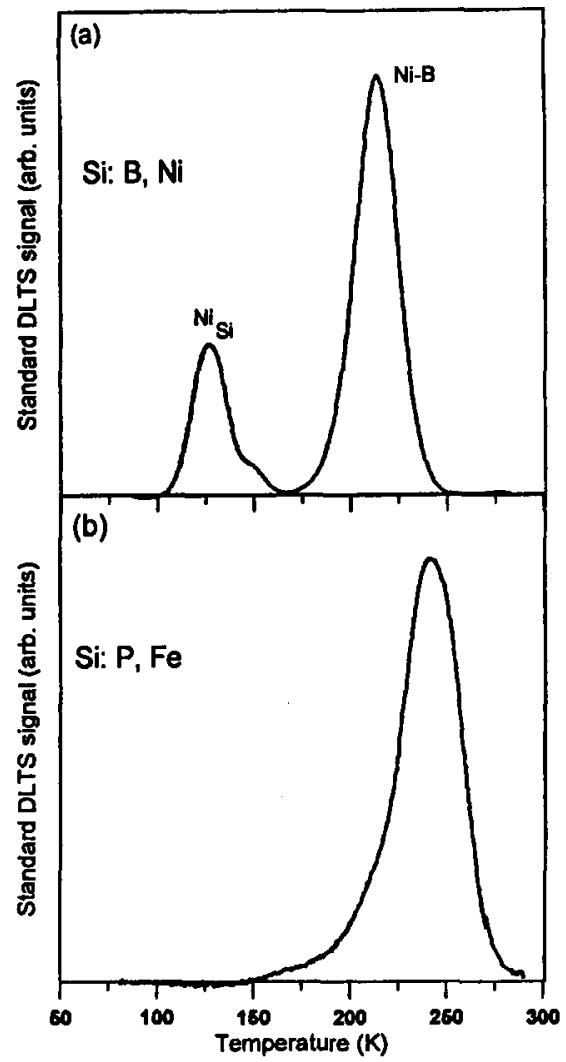

Fig. 1. Standard DLTS spectra of (a) $\mathrm{Ni}$ in $p$-type, and (b) Fe in $n$-type silicon.

In our previous studies we have demonstrated [1] that Laplace-transform DLTS offers at least one order of magnitude better resolution than the standard DLTS method. It allows us to observe the influence of small disturbances on the process of carrier thermal emission from defects. Alternatively, it allows also to distinguish defects with slightly different emission characteristics. In this paper a possible fine structure hidden in broad DLTS peaks, usually associated with the presence of TM impurities or thermal donors (TD) in the crystal, has been examined.

The samples used in our study were grown by the vapour phase epitaxy on $p^{+}$- or $p$-type (boron doped) substrates. The active layer was lightly doped with boron and covered by the $n^{+}$-type layer to form the $p-n$ junction. For the case of the $n$-type active layer the substrate was $n^{+}$-type and the top layer was $p^{+}$-type. Prior to the growth process the back surface of the substrate was intentionally contaminated by the $4 \mathrm{~N}$ purity $\mathrm{TM}$ foil ( $\mathrm{Co}, \mathrm{Cu}, \mathrm{Ni}, \mathrm{Pd}$, or $\mathrm{Fe}$ ). In each of the growth processes always one uncontaminated layer was grown in order to produce the reference sample. For the studies of the thermal donor fine structure the P-doped floating zone silicon crystals have been used. The silicon for TD studies 


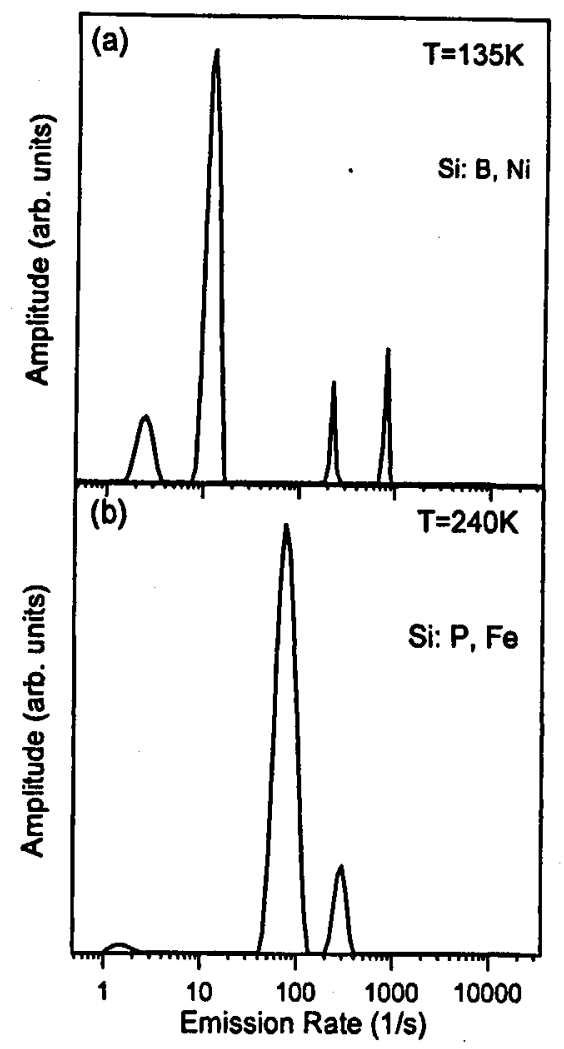

Fig. 2. Laplace-transform DLTS spectra (a) $\mathrm{Ni}$ in p-type (low-temperature peak in Fig. 1(a), and (b) Fe in $n$-type silicon.

was not subjected to any heat treatment, thus only residual (concentration smaller than $10^{12} \mathrm{~cm}^{-3}$ ) content of the thermal donors generated during crystal cooling process was observed. For this case the Au-Schottky diodes on the layer top have been fabricated.

For the case of the TM diffused samples the DLTS measurements revealed (by a comparison to reference samples) the appearance of defect centres which could be referred to the diffusion process. However, despite the fact that the standard DLTS shows no apparent structure for them (see Fig. 1), some of the TM related defects have a very similar emission characteristics to other not TM-related centres in silicon. For example, in $p$-type boron-doped crystals there is a number of defects having the activation energy for the hole emission between 0.18 and $0.25 \mathrm{eV}$. Among them the most typical are: divacancy, B-vacancy, and B-O complexes [2]. These defects produce DLTS peaks which usually overlap with the peaks related to some transition metal impurities, e.g., an isolated nickel centre (low-temperature peak in the spectrum shown in Fig. 1a). A similar phenomenon occurs in $n$-type silicon where the peak related to the P-vacancy complex coincides with the iron-related peak observed by us (Fig. 1b). For the peaks which could overlap with the peaks 
related to other defects the Laplace-transform DLTS method indeed revealed the well-resolved fine structure in the carrier thermal emission process. For example, the isolated nickel-related hole emission could be decomposed into four distinct components (see Fig. 2a), and for the iron-related emission in the $n$-type silicon the Laplace DLTS demonstrated two components (Fig. 2b). For the case of the thermal donors the DLTS signal related to two charge states of the defect could be observed. The emission process from the shallower state $(0 /+)$ with the ionization energy of $54 \mathrm{meV}$ could be investigated at temperatures between 35 and $40 \mathrm{~K}$. The emission from the deeper one $(+/++)$ (ionization energy $153 \mathrm{meV}$ ) was observed in the temperature range between 65 and $100 \mathrm{~K}$. The Laplace DLTS measurements for both cases show the structure of two or three lines. The observation of the TD fine structure is fully consistent with the fact that the thermal donors form a defect family [3]. This phenomenon being well known from the results obtained by using other experimental techniques was observed in this study by the DLTS method for the first time.

\section{References}

[1] L. Dobaczewski, P. Kaczor, I.D. Hawkins, A.R. Peaker, J. Appl. Phys. 76, 194 (1994).

[2] See, e.g., in: Landolt-Börnstein New Series III/22b, Ed. F. Shimura, Academic Press, Boston 1994, Ch. 4.2.3, p. 270.

[3] J. Michel, L.C. Kimerling, in: Semiconductors and Semimetals, Vol. 42, Eds. O. Madelung, M. Shulz, Springer-Verlag, Berlin 1989, p. 257. 